\title{
Student Perceptions of Adverse Health Events During Ambulance Clinical Placements
}

\author{
Jade Sheen", Leanne Boyd, Kathryn Eastwood, Frank Archer, Sinead Leaf \\ Department of Community Emergency Health and Paramedic Practice, Monash University, \\ Frankston, 3199, Australia
}

\begin{abstract}
This pilot study intended to augment current literature in the clinical placement field by investigating the frequency and nature of adverse health events experiences by paramedic students undertaking ambulance clinical placements. Supports accessed post event were also reviewed. A purposive sample of fifty-six paramedic students completed the questionnaire. The results indicate that a number of students experience adverse health events while on clinical placement, with fourteen cases of verbal abuse, one case of physical abuse, nine cases of sexualised behavior and seven cases of psychological distress reported. While some case related incidents were flagged by ambulance services and followed up by peer support, students did not initiate any formal support processes themselves. Moreover, no student filed a formal report regarding any of the incidents raised. The results of this pilot study require further investigation. In the interim, the benefits of clinical placements must be weighed against their risks, and processes put in place to minimize the risk to students undertaking clinical placements.
\end{abstract}

Keywords Occupational Health and Safety, Clinical Placements

\section{Introduction}

What it is that constitutes effective teaching practice is a contentious issue, particularly in the health and medical disciplines. There does appear to be some consensus however, regarding the importance of field experience to augment teaching and learning (1). Research indicates that professional socialisation and concepts such as 'reality shock' are addressed when students have the opportunity to work as a peer in their chosen profession(2). This is especially important in the pre-hospital field where a high stimulus environment which is not found in a university, can greatly effect treatment decisions and patient outcome (1).

Within contemporary paramedic practice, field experience is commonly referred to as 'clinical placement'. Unfortunately, there are a number of issues attached to the undertaking of clinical placement in Australia. There are limitations in the number of placements available, with an increasing student market and fewer experienced clinicians available supervise students. In addition, the quality of the placement experience has been variable. Anecdotally, concerns have also been raised regarding more serious issues that may occur during paramedic clinical placements, in cluding exposure to verbal and physical abuse. It is the latter issue that becomes the focus of this paper.

* Corresponding author:

jade.sheen@monash.edu (Jade Sheen)

Published online at http://journal.sapub.org/edu

Copyright (C) 2012 Scientific \& Academic Publishing. All Rights Reserved

\section{Literature Review}

Boyle et al (2008) conducted one of only a few studies of the experiences of undergraduate students during paramedic clinical placement (3). Fifty five percent of the participants surveyed reported that they were not made to feel welcome during placement, while $57 \%$ reported that at least one crew member treated them with disdain. Concerns regarding the physicality of job and students ability to take on this aspect of the job were also raised. Despite these reports, $93 \%$ of participants felt that the clinical placement experience was still a positive one, highlighting the perceived inherent value of this type of learning. The authors of this study raised concerns regarding the impact of these reports on students and their learning.

While not focussing on the student experience specifically, Petzall at el. examined the experiences of Swedish nurses and paramedics in the prehospital environment (4). They found that $66 \%$ of respondents had experienced threats or violence in the course of their work experience. An alarming $26 \%$ of participants had been threatened and $16 \%$ had been physically attacked in the 12 months prior to the study. In contrast with the findings from Boyle et als study, Petzall found no correlation between gender, experience or position on the likelihood of adverse occupational health events occurring $(3,4)$. Other studies examining the experiences of paramedics in the prehospital environment suggest that verbal abuse (5), occupational violence (6-14) and sexual harassment (5) may occur. As paramedic students work in the same environment as the qualified paramedics surveyed, it stands to reason that students are exposed to similar events. 
Research indicates similar difficulties occurring in related health and medical fields. For example, Curtis, Bowen and Reid investigated nursing student's experiences of horizontal violence on clinical placement (15). Horizontal violence is defined within the literature as interpersonal conflict among peers (16). The researchers found that $57 \%$ of students had experienced horizontal violence while on clinical placement, with $90 \%$ indicating that these experiences would impact on their future career choices. More disturbingly, some participants named staff members as the perpetrators of violence. While this study was limited in that it had a very small sample size and a low response rate, with participants potentially excluding themselves from the study if they did not have any experience of violence to report, even one case is worthy of further investigation by educational institutions as the protection of students is paramount. Other concerns regarding negative experiences while on clinical placement, including physical injury and manual handling $(17,18)$, violence $(19,20)$ and bullying $(15,16,21-23)$, have been reported in nursing allied health fields. While these studies were not paramedic specific, it is reasonable to assume that there may be some parallels given the nature of the work encountered.

The present study aims to overcome the limitations of previous research by conducting a more comprehensive, albeit preliminary, investigation into adverse occupational health and safety events during paramedic clinical placements. Specifically, this study aims to identify the frequency and nature of different adverse health events that may occur during paramedic clinical placements. For the purpose of this study, the term adverse health event includes exposure to verbal and physical abuse, injury, sexualised behaviour and emotional and psychological distress. Actions taken in the event of an adverse health event will also be identified.

\section{Method}

Ethics committee approval was obtained before the commencement of any data collection.

\subsection{Sampling Method}

A purposive sample of paramedic students undertaking clinical placement as a component of their coursework was utilised.

\subsection{Measures}

Participants completed a written questionnaire with two components.

Section A, contained demographic data including participants' gender, age range, the degree they were enrolled in and their year of study.

Section B, contained quantitative data and a limited qualitative component. This section was designed to identify any trends or concerning situations occurring during clinical placements. Section B asked participants to identify if they had experienced any of the following during their ambulance clinical placements:
- Verbal (e.g. yelling, swearing or bullying) abuse;

- Physical abuse (e.g. pushing, hitting, kicking or being hit by a projectile, weapon or other object);

- Sexualised behaviours (e.g. overt sexualised commentary, touching or kissing);

- Physical illness or injury; and/or,

- Emotional distress (e.g. extreme stress, uncontrollable worry, lowered mood, nightmares, flashbacks of significant events, etc).

Participants confirming any of these experiences were then asked whether the alleged perpetrator was a staff member, patient, family member or bystander. Participants were also asked whether they accessed peer support or filed any reports regarding the incident in question and whether they have experienced any ongoing distress following the incident. In the case of the last two variables, physical illness/injury and emotional distress, participants were also to specify the nature of the injury or impact.

\subsection{Procedure}

1. Volunteers were recruited at the commencement of university lectures and at student paramedic forums.

2. Those agreeing to participate were provided with consent forms, Plain English Statements and questionnaires, to be completed and posted to the researchers. While this method is often associated with poor response rates, providing mail boxes close to the site of distribution and return paid envelopes resulted in a final response rate of seventy six percent.

3. Quantitative questionnaire data was collated and processed using SPSS.

\section{Results}

Descriptive and frequency data were analysed using SPSS Version 18. While this basic level of analysis has its limitations, it does allow for preliminary observations of trends and experiences, which is consistent with the stated aims of the study. When examining the report data below, it is important to note that in some cases, individual participants made multiple reports under one or more categories.

\subsection{Participants}

Fifty six university students, 22 males and 34 females, participated in the survey. This represented an $89 \%$ response rate for those present at the time of recruitment. Approximately $43 \%$ of the cohort were in their third year their undergraduate paramedic degree, $29 \%$ were accelerated entry paramedic students, while $27 \%$ were in the final year of a double degree Nursing/Paramedic course. One participant failed to nominate which degree they were completing. All participants had undertaken at least 20 days of clinical placement within an Ambulance service. Participants represented a large age range, with five participants aged 18-20 years; forty-one aged 21-25 years; seven aged 26-30 years; two aged 31-40 years and one aged 41 years or over. 
Jade Sheen et al.: Student Perceptions of Adverse Health Events During Ambulance Clinical Placements

Table 1. Number of reports made based on category

\begin{tabular}{|c|c|c|c|c|}
\hline $\begin{array}{c}\text { Verbal } \\
\text { abuse }\end{array}$ & $\begin{array}{c}\text { Physical } \\
\text { abuse }\end{array}$ & $\begin{array}{c}\text { Sexualised } \\
\text { behaviour }\end{array}$ & $\begin{array}{c}\text { Emotional } \\
\text { distress }\end{array}$ & $\begin{array}{c}\text { Physical ill- } \\
\text { ness or injury }\end{array}$ \\
\hline 14 & 1 & 9 & 7 & 3 \\
\hline
\end{tabular}

Fourteen of the fifty-six respondents reported some form of verbal abuse during their ambulance clinical placements. Of these fourteen reports, nine of the identified perpetrators were patients while five were ambulance service staff. While many participants reported that debriefing was made available to them, none followed up on this option and no students filed a report on the matter.

One incident of physical abuse was reported, though the participant in question qualified that this occurred in the context of a patient seizure and was thus unintentional.

Nine of the fifty-six respondents reported episodes of sexualised behaviour. Four incidents were perpetrated by patients and five by staff. Peer support was not accessed by any of the participants reporting these incidents and no reports were filed.

Seven of the fifty-six respondents reported experiencing some form of emotional or psychological distress during paramedic clinical placements. Of these incidents, two were directly related to bullying perpetrated by ambulance service staff who were directly responsible for the student on placement (other participants reported similar experiences under the 'verbal abuse' category), one was related to unprofessional behaviour of staff members including failure to follow clear ambulance service policies and guidelines, three were related to patient deaths and/or trauma and one was related to verbal abuse perpetrated by a patient. In each case students reported being adversely affected by the incident for a period of time. Cases that related to patient deaths were followed up by peer support, with students reporting that the process was helpful. No support was accessed when peer support was not aware of the situation.

Three incidents of physical illness and/or injury were reported. One case of back strain, one cut requiring sutures and one case of flu was reported. No reports were filed.

\section{Discussion}

As there is a dearth of literature directly relating to this topic, the present study was undertaken as a pilot to identify if adverse health events are indeed occurring, the frequency and nature of such events and whether any supports were accessed or reports filed in response.

The results indicate that a quarter of students undertaking paramedic clinical placements experience some form of verbal abuse during the placement itself, with one reporting actual physical abuse, though this was in the context of a medical condition with no intent or malice associated. These findings reflects to a lesser degree, those of by Petzall et al., who noted $66 \%$ of nurses and paramedics in the prehospital environment experienced some form of threat, violence or intimidation while working (4). Boyle, Coritsas and Coles (2006) report similar findings, with the majority of qualified
Ambulance Paramedics surveyed experiencing some form of threat or violence in the preceding 12 months (5). What actions can be taken to address this issue with students however, remains unclear. While we can assist in the provision of de-escalation skills to assist students when faced with aggressive patients and encourage a general awareness of danger and safety issues, we cannot completely ameliorate the potential for verbal abuse or intimidation.

Students in the present study, who reported exposure to verbal abuse, noted that debriefing was the most useful tool available to help them process the situation, with the majority reporting that they discussed the case with the paramedic crew after the incident. The benefits of immediate support from peers who shared the situation cannot be underestimated. Qualified Paramedics should take this into account when supervising students. While it is not always possible to avoid swearing or other forms of verbal abuse from patients or bystanders, supervising paramedics can help students to process what they encountered and make sense of it. Studies provide some support for this claim, suggesting that reframing incidents and attempting to understand them can protect against more significant forms of emotional distress, such as Post Traumatic Stress Disorder (PTSD) (see 24 for a review).

Nine of the students surveyed also reported experiencing some form of sexualised behaviour while undertaking ambulance clinical placements. Five of the nine cases were perpetrated by qualified staff, the remaining four by patients. While they appear alarming, these findings should be interpreted with caution, as participants were not asked to qualify their responses or provide a context. They were simply asked to identify if they had experienced any sexualised behaviours towards them during their ambulance clinical placements (e.g. overt sexualised commentary, touching, kissing, etc). In the absence of any qualitative feedback or formal reporting by students we can only stipulate that this finding warrants further attention.

With regards to the emotional distress aspect of the study, the results indicated that only seven of the fifty-six respondents experienced an event(s) that lead to them feeling distressed. The low numbers of students reporting distress is in some ways surprising, as death across the lifespan, as well as access to some graphic trauma scenes, appears to be an inherent component of ambulance practice. This finding may be linked with the fact that when difficult jobs occur there is a clear pathway for support, with peer support being activated without the student necessarily requesting the support. Paramedic crews may also be more likely to identify this aspect of the work as difficult and therefore may implement some form of informal debriefing or support after the job has finished. This is distinct from the processes for other forms of adverse health events, which tend to rely on the students to access support or activate the reporting procedure. Students may also be reluctant to report on issues such as bullying or verbal abuse, due to a power imbalance between students and qualified staff or fears that reporting may have ramifications for their career progression or employment prospects. 
With regards to emotional distress, it is important that educators prepare students for the possibility before clinical placements commence, normalising emotional responses where appropriate and discussing management of strong emotions. Support should also be made available during and after the placement period, accounting for immediate or delayed responses to any event(s) encountered. Encouraging education and awareness of mental health issues among paramedic students is an important step in reducing the high rates of anxiety, depression, PTSD and suicide that have been noted among qualified staff (25-33). Introducing the concept of support and education early on, may also lead to a gradual change in the culture of the paramedic workforce, encouraging access to peer support and associated supports such as the Victorian Ambulance Crisis Counselling Unit.

The illness and injury data is not unforseen as there are obviously exposure risks and hazards associated with the prehospital environment $(34,35)$. While AV have a number of policies in place to decrease the risk and Universities teach optimal occupational health and safety practices prior to the commission of any placement, these issues may still occur. The most concerning aspect of these findings from a teaching perspective, is that no reports or formal documentation were filed after the events. This is obviously very concerning as without formal documentation, the health and rights of our students cannot be ensured.

The findings discussed indicate that students are being exposed to adverse health events while undertaking paramedic clinical placements. While measures have been put in the place within the University to support students, no intervention will be effective if it is not being accessed. Understanding the barriers to accessing support, debriefing or reporting is therefore crucial. Future research should focus on the barriers to accessing support.

The results of this study are limited by the small sample size. This study was designed to identify in a more formal sense, whether adverse occupational health events were occurring during paramedic clinical placements as there had been some anecdotal reports of such issues. While the number of students experiencing adverse health events was not overwhelming, it could be argued that any student experiencing issues like bullying, verbal abuse or sexualised behaviour in the workplace should be supported. As such, further study and analysis is warranted.

\section{Conclusions}

This paper has identified the frequency and nature of different adverse health events that have occurred during paramedic student clinical placements. The importance of clinical placements to students has been supported within the literature and the findings of this study. While clinical placement opportunities may be limited and can be a challenge for ambulance services to arrange, students response to them is generally very positive, with students reporting that placements allow them to access skills and learning opportunities that cannot be easily replicated in the classroom environment. These positives need to be weighed against the difficulties that may occur as a number of students are experiencing adverse health events such as verbal abuse, sexualised behaviour and bullying, while on paramedic clinical placement. Of more concern is the fact that few students' access peer support or formal debriefing when these events occur and no reports have been filed which would allow for ambulance services or universities to act on the events. Students obviously need to be prepared for the challenges they may face during placements, supported during and after the placement block and informed of their rights and protections should an adverse health event occur.

\section{ACKNOWLEDGEMENTS}

Many thanks to those who chose to participate in this survey.

\section{REFERENCES}

[1] Crossman. Technical and environmental impact on Medication error in Paramedic Practise: A review of causes, consequences and stratigies for prevention. JEPHC. 2009;7(3).

[2] McKenna LG, Wray N, McCall L. Exploring continuous clinical placement for undergraduate students. Advances in Health Sciences Education. 2009;14(3):327-35.

[3] Boyle M, Williams B, Cooper, Adams, Alford. Ambulance clinical placements- A pilot study of students' experience. BMC Medical Education. 2008;8(9):1-6.

[4] Petzäll K, Tällberg J, Lundin T, Suserud B-O. Threats and violence in the Swedish pre-hospital emergency care. International Emergency Nursing. In Press.

[5] Boyle, Koritsas, Coles. Perpetrators of violence against paramedic's in the workplace and the paramedic's responce. JEPHC. 2006;1(1):1.

[6] Brough P. Workplace violence experienced by paramedics: Relationships with social support, job satisfaction, and psychological strain. Australasian Journal of Disaster and Trauma Studies. 2005;2:1-12.

[7] Corbett S, Grange J, Thomas T. Exposure of prehospital care providers to violence. Prehospital Emergency Care. 1998: 127-231.

[8] Grange J, Corbett S. Violence Against Emergency Medical Services Personnel. Prehospital Emergency Care. 2002;6(2): 186-90.

[9] Hafeez S. The relationship of violence-related trauma and length of trauma exposure to Post-Traumatic Stress Disorder in Emergency Medical Services personnel. Dissertation Abstracts International: Section B: The Sciences and Engineering. 2003;63(11 B):5517.

[10] Koritsas S, Boyle M, J. C. Factors Associated with Workplace Violence in Paramedics. Prehospital \& Disaster Medicine. 
2009; 24(5):417-21.

[11] Lucas R. Violence in the prehospital setting. Emergency Medical Clinics of North America. 1999; 17:679-83.

[12] Mayhew C, Chappell D. Ambulance officers: the impact of exposure to occupational violence on mental and physical health. The Journal of Ocupational Health and Safety Australia and New Zealand. 2009; 25(1):37-49.

[13] Pozzi C. Exposure of prehospital providers to violence and abuse. Journal of Emergency Nursing. 1998; 24(4):320-3.

[14] Suserud BO, Blomquist M, Johansson I. Experiences of threats of violence in the Swedish ambulance service. Accident and Emergency Nursing. 2002; 10: 127-35.

[15] Curtis J, Bowen I, Reid A. You have no credibility: nursing students' experiences of horizontal violence. Nurse Education in Practice. 2007; 7(3):156-63.

[16] Duffy E. Horizontal violence: a conundrum for nursing. Journal of the Royal College of Nursing Australia. 1995;2(2):5-9.

[17] Kneafsey R, Haigh C. Learning safe patient handling skills: student nurse experiences of university and practice based education. Nurse Education Today 2007; 27(8):832-9.

[18] Glover W, McGregor A, Sullivan C. Work-related musculoskeletal disorders affecting members of the chartered society of physiotherapy. Physiotherapy (London). 2005; 91(3):138-47.

[19] Ellwood AL, Rey LD. Awareness and fear of violence among medical and social work students. Workplace violence towards social workers: The Australian experience. Family Medicine. 1996; 28(7):488-92.

[20] Hinchberger PA. Violence against female student nurses in the workplace. Nursing Forum. 2009; 44(1):37-46.

[21] Longo J. Horizontal Violence among Nursing Students. Archives of psychiatric nursing. 2007; 21(3):177-8.

[22] Physiotherapy. CSo. Dealing with bullying: a guide for physiotherapy students on clinical placement;; [cited 2011]; Available from:

http://www.csp.org.uk/publications/dealing-bullying-guide-p hysiotherapy-students-clinical-placement.

[23] Hoel H, Giga SI, Davidson MJ. Expectations and realities of student nurses' experiences of negative behaviour and bullying in clinical placement and the influences of socialization processes. Health Services Management Research. 2007; 20(4):270-8.
[24] Foa EB, Keane TM, Friedman MJ. Guidelines for treatment of PTSD. Journal of Traumatic Stress. 2000; 13(4):539-88.

[25] Lowery K, Stokes MA. Role of peer support and emotional expression on posttraumatic stress disorder in student paramedics. Journal of Traumatic Stress. 2005; 18(2):171-9.

[26] Smith A, Roberts K. Interventions for post-traumatic stress disorder and psychological distress in emergency ambulance personnel: a review of the literature. Emergency Medicine Journal. 2003; 20(1):75-8.

[27] Gallagher S, McGilloway S. Experience of critical incident stress among ambulance service staff and relationship to psychological symptoms. International Journal of Emergency Mental Health. 2009; 11(4):235-48.

[28] Grevin F. Posttraumatic stress disorder, ego defense mechanisms, and empathy among urban paramedics. Psychological Reports. 1996; 79(2):483-95.

[29] Beaton RD, Murphy S, Johnson C, al. e. Exposure to duty-related incident stressors in urban firefighters and paramedics. Journal of Traumatic Stress. 1998; 11:821-8.

[30] Holland W. Fire/emergency medical services and coping methods: Mitigating traumatic stress symptomatology in emergency services professionals. Virginia: Liberty University; 2008.

[31] McCammon SL. Emergency medical service workers: occupational stress and traumatic stress. In: Paton D, Violanti JM, editors. Traumatic stress in critical occupations: recognition, consequences and treatment. Springfield: Charles C Thomas; 1996. p. 58-86.

[32] Mitchell JT, Dyregrov A. Traumatic stress in disaster workers and emergency personnel: prevention and intervention. In: Wilson JP, Raphael B, editors. International handbook of traumatic stress syndromes. New York: Plenum Press; 1993. p. $905-14$.

[33] Spencer WM. Peritraumatic dissociation and posttraumatic symptomatology in urban paramedics. Dissertation Abstracts International: Section B: The Sciences and Engineering. 2006; 67(3-B):1718.

[34] Donnelly E, Siebert D. Occupational risk factors in the emergency medical services. Prehospital \& Disaster Medicine. 2009; 24(5):422-9.

[35] Maguire BJ, Hunting KL, Smith GS, Levick NR. Occupational fatalities in emergency medical services: a hidden crisis. Annals of Emergency Medicine. 2002; 40(6):625-32. 